\title{
Universal digital strain gauge measurement system of aeroelastic deformation development
}

\author{
Anton Pavlov ${ }^{1, a}$ and Alexander Arefiev ${ }^{1}$ \\ ${ }^{1}$ Saint-Petersburg University of Aerospace instrumentation, 190000 Saint-Petersburg, Russia
}

\begin{abstract}
This article presents description of the universal digital strain gauge system developed to measure the static and dynamic aeroelastic deformations of elasticity-scale models during the tests in aerodynamic tube and during in-flight tests of an experimental air vehicles. The main requirements for such devices are small size and possibility of operation in a wide temperature range. The article considers the dependence of zero offset from temperature. Functional diagram block and logic diagram of the build system are shown.
\end{abstract}

\section{Introduction}

Today the great attention is paid to concept investigation of an active aeroelastic wing. This concept allows to improve maneuvering characteristics for a wide range of speed, angles of attack, heights and at the same time reducing weight of the aircraft [1].

The synthesis of the control system within the concept of aeroelastic wing necessarily requires an application of the principle of integrated design, in which all the links of the chain: design aerodynamics - strength are related. Control system of aeroelasticity requires high attention to the accuracy of determination of parameters of the aircraft in the wind tunnel tests, including elasticityscale models. Characteristics of elasticity of the studied objects during wind tunnel and in-flight tests are basically defined with the help of strain gauge measurements.

This article presents the concept of universal digital strain gauge system that can be installed on elasticity-scale models for wind tunnel tests and in experimental air vehicles [2].

Thus, the main system requirements are: miniaturization of its components, and possibility of its operation in a wide temperature range. In addition, measurement components of this system could be connected by wired connection or/and wireless.

\section{Description of system}

The described strain gauge system allows taking date simultaneously from not less than 50 strain gauge measuring units, and they could be connected by wired and/or wireless way.

\footnotetext{
${ }^{a}$ Corresponding author : sa807@bk.ru
} 


\section{MATEC Web of Conferences}

\subsection{Structure of system}

System consists of: conversion unit USB-RS485; radio transmit-receive unit; signals distribution unit; strain measuring force unit; strain measuring deformation unit; extender interface.

All system components have a standard interface RS485 and power 6 to $25 \mathrm{~V}$, and input and output interface that allows to connect blocks in parallel to signals distribution unit or sequentially to each other, and also through the radio transceiver unit. Figure 1 shows an example of connecting the measuring units in a common measurement system and collection of information that can be installed on the investigated object.

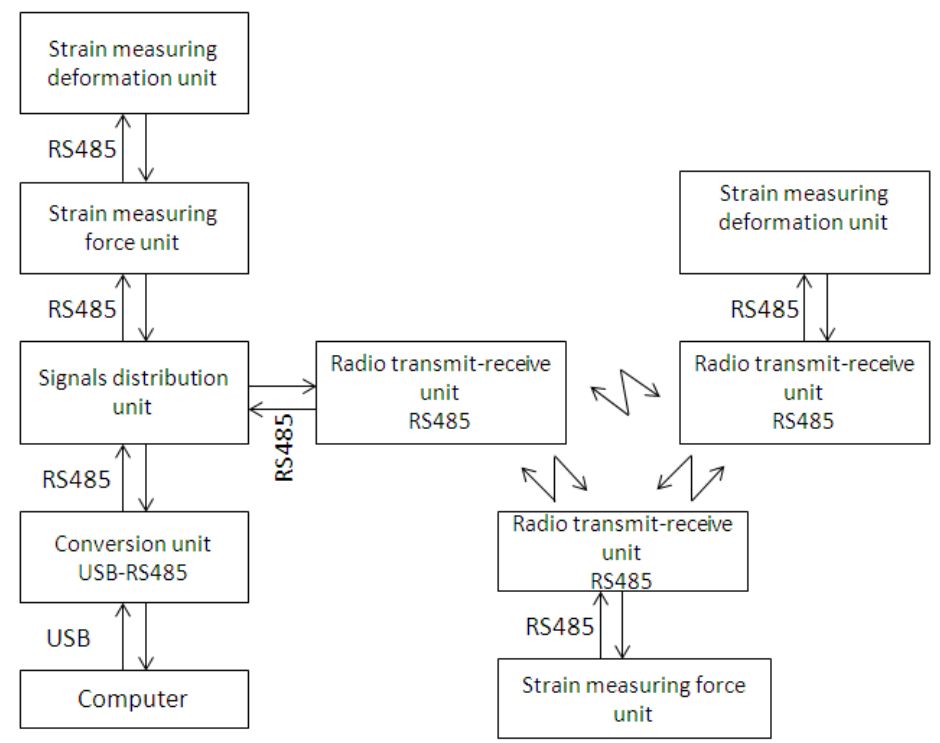

Figure. 1. Structural diagram of connection blocks in a single system designed for installation on elasticity-scale model.

\subsection{Strain measuring force unit}

Strain gauge in this unit is used to measure the force. As a sensitive element it uses strain gauge measuring pressure with strain-gage bridge. Structural diagram of the strain measuring force unit is shown in Figure 2. This unit is made with modular design and allows to use a large variety of strain gauges [3].

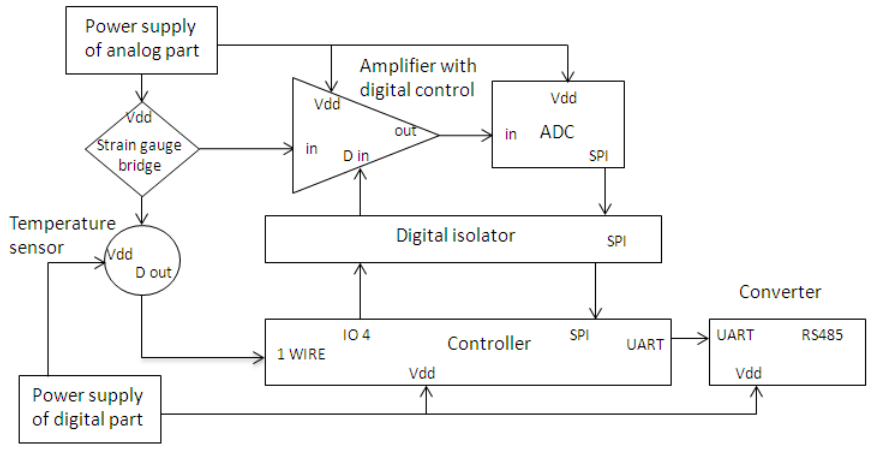

Figure 2. Structural electric diagram of the strain measuring force unit. 
Strain measuring force unit can be divided into two parts: analog and digital. Analog part consists of: power supply of analog part; strain gauge bridge; amplifier with digital control; ADC. There is a low-pass filter at the output of the strain gauge. The amplifier converts the analog signal from the strain gauge to the signal from 0 to $3.3 \mathrm{~V}$. Amplifier is equipped with a digital control zero offset and scale factor. ADC converts analog signal to digital and transmits it to the controller by SPI interface.

Digital part consists of: controller; power supply of digital part; temperature sensor; digital isolator; converter USB-RS485.

Digital isolator is used for galvanic isolation of analog part from digital, to reduce the noise effect generated by digital parts to the power supply of analog part. The temperature sensor measures the temperature of the strain gauge and circuit pack. It should be noted that each strain measuring force unit is previously calibrated in a climatic chamber at the required temperature range. The aim of calibration is the determination of scaling factors of the strain gauge and zero offset in the operating temperature range.

Thus, the temperature sensor serves to eliminate the influence of temperature on measured parameters of the strain gauge. The controller reads the data, filters, processes the request, and sends to the consumer via converter USB-RS485.

The firmware of the strain measuring force unit provides: setting of the amplifier; reading of ADC parameters; reading the data from the temperature sensor; filtering the data; conducting of compensation of bias and scale temperature factor; receiving and processing external commands; recording calibration data. Such implementation simplifies the work with strain gauge sensor and reduces the amount of post-processing.

\subsection{Strain measuring deformation unit}

Strain measuring deformation unit is used to measure elastic deformations, arising under tensioncompression, bending and torsion. As a sensitive element in this unit is used strain-gauge plate mounted by special glues. If the test objects are held under high humidity for a long time, the sensitive elements are protected by a moisture-resistant coatings. The electronic part of this is completely similar to the strain measuring force unit. The main difference in comparison with strain measuring force unit is using another type of sensitive element, method of its calibration and processing sensor data. This unit also enables the connection a wide variety of analog sensors.

\subsection{Measuring oscillation unit}

One of the most important areas of investigations of dynamic characteristics of aeroelastic oscillations is their bodies and control systems. These studies enable to obtain extremely valuable information about the effectiveness of combating with flutter and buffeting. The basis of the proposed strainmeasuring units can be used as the unit for measuring the frequency characteristics of the test objects. For this purpose, it is necessary to increase the frequency of removal of parameters of strain measuring force unit, connect the accelerometer as a sensitive element and change its firmware.

\section{The layout of the strain measuring force unit}

The layout of the strain measuring force unit is made of elements represented in the Figure 2. The layout is connected to personal computer through USB, for transmission of processed force and temperature data for further processing in it. The layout of the strain gauge measuring force unit is shown in Figure 3. Making layout pursuit the aim to test the circuitry of this block, as well as studies of the temperature effect on zero offset of the strain gauge, and to determine the proper allocation of the temperature sensor. Figure 4 shows a graph of zero offset from temperature. It should be noted, that the layout is designed in such a way that allows connecting a wide range of strain gauges. 


\section{MATEC Web of Conferences}

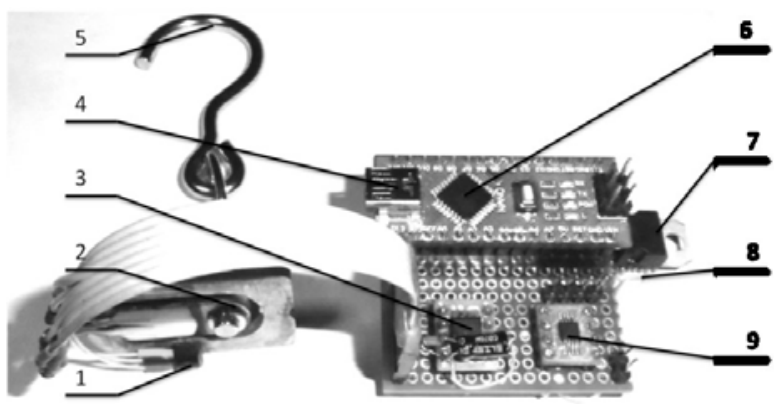

Figure 3. The layout of the strain gauge measuring unit of force. 1 - Temperature sensor, 2 - strain gauge, 3 - amplifier, 4 - USB connector, 5 - hook of strain gauge, 6 - controller, 7 - analog power supply, 8 digital isolator, $9-\mathrm{ADC}$.

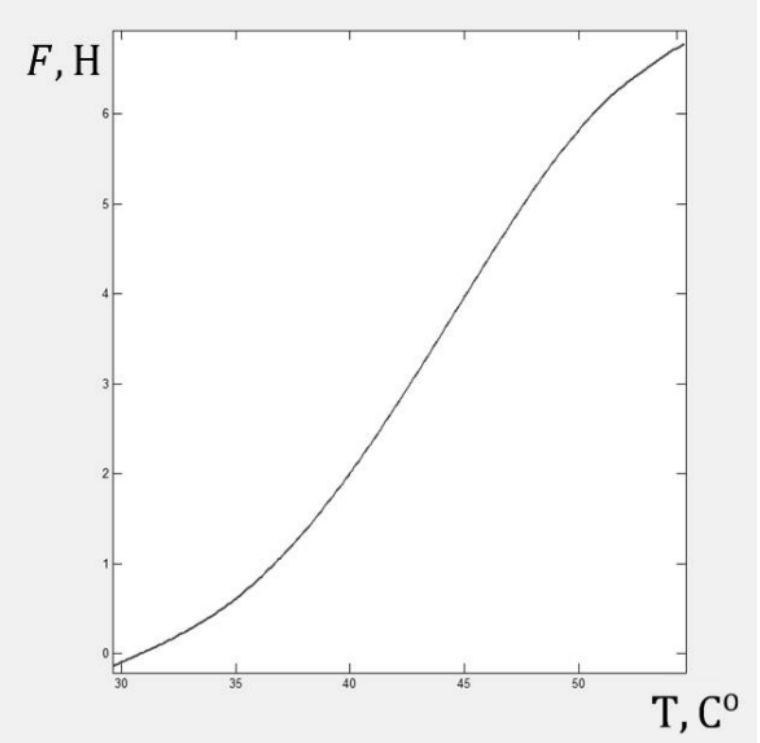

Figure 4. A graph of the dependence of zero offset from temperature.

\section{Conclusion}

This article presents the concept of a universal digital strain measurement system of aeroelastic deformations. Structural system diagram and layout of the strain gauge measuring force unit were introduced. In addition, a temperature effect on zero offset of the strain gauge was shown.

The prototyping of components of the system is going to reduce the dimensions of each unit. Thus, it will be possible to ensure the installation of the proposed system on elasticity-scale models, which are used in wind tunnel tests and experimental air vehicles.

\section{References}

1. Campanile, L. F. and Sachau, D. J. Intell. Mater. Syst. Struct. 10, 215 (2000)

2. J. R. Wright, J. E. Cooper, Introdaction to Aircraft Aeroelasticity and Loads, ISBN 978-047085840-0 (2007)

3. M. Jafaripanach, B. M. Al.-Hashimi, N. M. White, IEEE International Symposium on Circuits and systems 4, IV 752 (2003) 